\title{
Hiperostosis esquelética difusa idiopática, a propósito de un caso
}

\author{
A. Espina Castilla, J. Forcada Gisbert*, X. J oaniquet Suils* \\ Médico residente MFyC. *M édicos especialistas
}

de MFyC. ABS 4. Badalona. Barcelona

\section{RESUMEN}

La hiperostosis esquelética difusa idiopática (DISH) es una patología cuya prevalencia aumenta con la edad, y que se caracteriza por una tenden cia a la osificación de tendones, ligamentos, pe riostio y cápsulas articulares, tanto espinales como extraespinales. Su diagnóstico y manejo terapéuti co debe ser realizado por el médico de familia.

Las manifestaciones radiológicas son su tarjeta de visita, aunque no hemos de olvidar sus manifes taciones clínicas espinales o extraespinales.

Derivaremos al segundo nivel asistencial en ca so de duda diagnóstica o cuando sospechemos patología compresiva (a nivel medular o esofágico).

El caso clínico que presentamos lo considera mos ejemplarizante por sus manifestaciones radio lógicas y clínicas.

Palabras clave: Hiperostosis esquelética difusa idiopática. Radiología. Clínica. Atención Prima ria.
Diffuse idiopathic skeletal hyderosis, a case report
Key words: Diffuse idiopathic skeletal hype rostosis. Radiology. Clinical Medicine. Primary Health Care.

\section{INTRODUCCIÓN}

La hiperostosis esquelética difusa idiopática (DISH) es una enfermedad ósea difusa que se manifiesta por una tendencia a la osificación de los ligamentos, tendones, periostio y cápsulas articulares, que afecta tanto a la columna vertebral como a estructuras extraespinales.

En 1950 Forestier y Rotés-Querol publican una serie de 9 casos donde describen la hiperostosis an- quilosante vertebral senil y la distinguen como entidad nosológica de la espondiloartrosis y la espondilitis anquilosante ${ }^{1}$. En 1956 Forestier describe las localizaciones periféricas, perdiendo el calificativo de vertebral.

El posterior hallazgo de casos entre los 40-50 años hizo suprimir el calificativo de senil.

No obstante forma parte de las cuatro enfermedades del envejecimiento articular junto a la osteoporosis, artrosis y condrocalcinosis. 
En 1975 Resnick et al publican un extenso estudio de las localizaciones extraespinales y nos muestran su elevada prevalencia ${ }^{2}$. Proponen el nombre de $D i$ fusse Idiopathic Skeleletal Hiperostosis (DISH).

El médico de familia la hallará radiologicamente en el $12 \%$ de los varones mayores de 70 años y en el $6 \%$ de los mayores de $40^{3}$. El diagnóstico diferencial se debe realizar con la osteoartrosis y la espondilitis anquilosante no sólo por rigor diagnóstico, sino por que tiene implicaciones terapéuticas.

A continuación presentamos un caso de DISH que destaca por la exuberancia de las manifestaciones radiológicas y por haber presentado manifestaciones clínicas.

\section{CASO CLÍNICO}

Varón de 72 años con antecedentes patológicos de obesidad (IMC>30) que consultó por limitación de la movilidad a nivel de la región cervical y que la manifestaba como imposibilidad para girarse o mirar al cielo. No refería dolor ni limitación de la movilidad en cintura escapular o pélvica.

En la exploración de la columna se objetivó una limitación de la movilidad en la región cervical y lumbar (flexión, extensión, rotación e inflexiones laterales).

Se solicitó radiografía de columna cervical (Fig. 1), dorsal (Fig. 2), lumbar (Fig. 3) y pelvis (Fig.4) y analítica que mostró: hemograma: hemoglobina 16,6 g/dl, hematócrito: 38,4\%, VCM: 91,5 fl, leucocitos $4,5 \times 10^{3} / \mathrm{ml}$, plaquetas $163 \times 10^{3} / \mathrm{ml}$, VSG: $12 \mathrm{~mm} / 1^{\text {a }}$ hora. Bioquímica: glucemia: $100 \mathrm{mg} / \mathrm{dl}$, triglicéridos: $140 \mathrm{ml} / \mathrm{dl}$, ac. úrico: 3,9 mg7dl, GOT: $13 \mathrm{U} / \mathrm{l}, \mathrm{GPT}: 7$ U/l, GGT: $16 \mathrm{U} / \mathrm{l}$ y F. alcalinas: $110 \mathrm{U} / \mathrm{l}$.

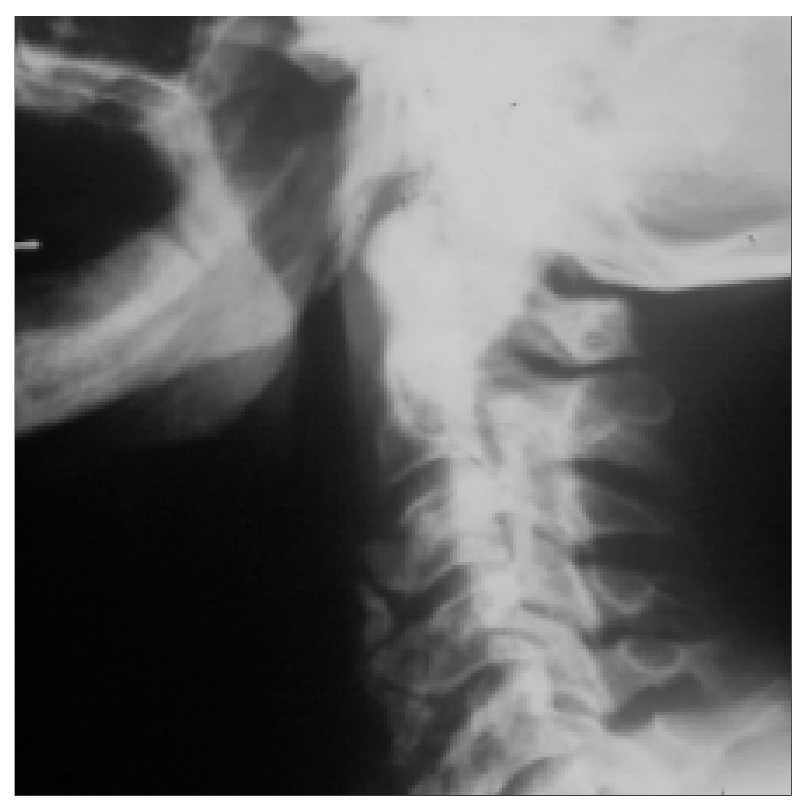

Figura 1

Hiperostosis exhuberante columna cervical.

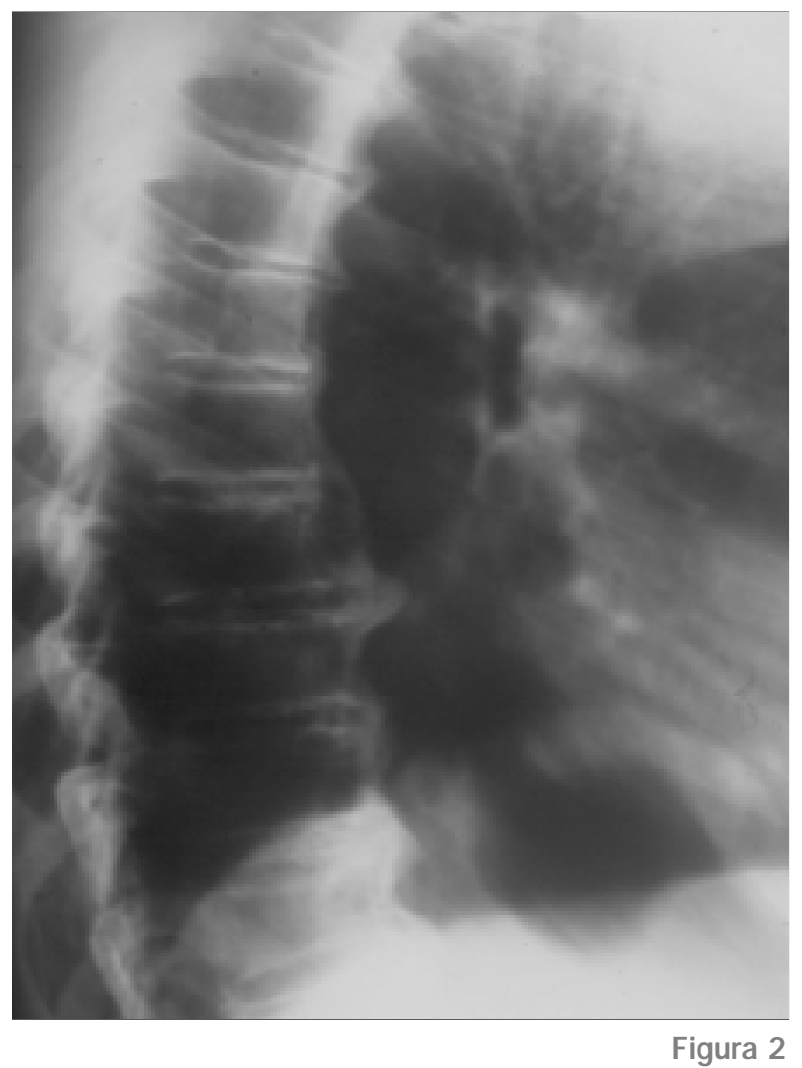

Hiperostosis en región dorsal.

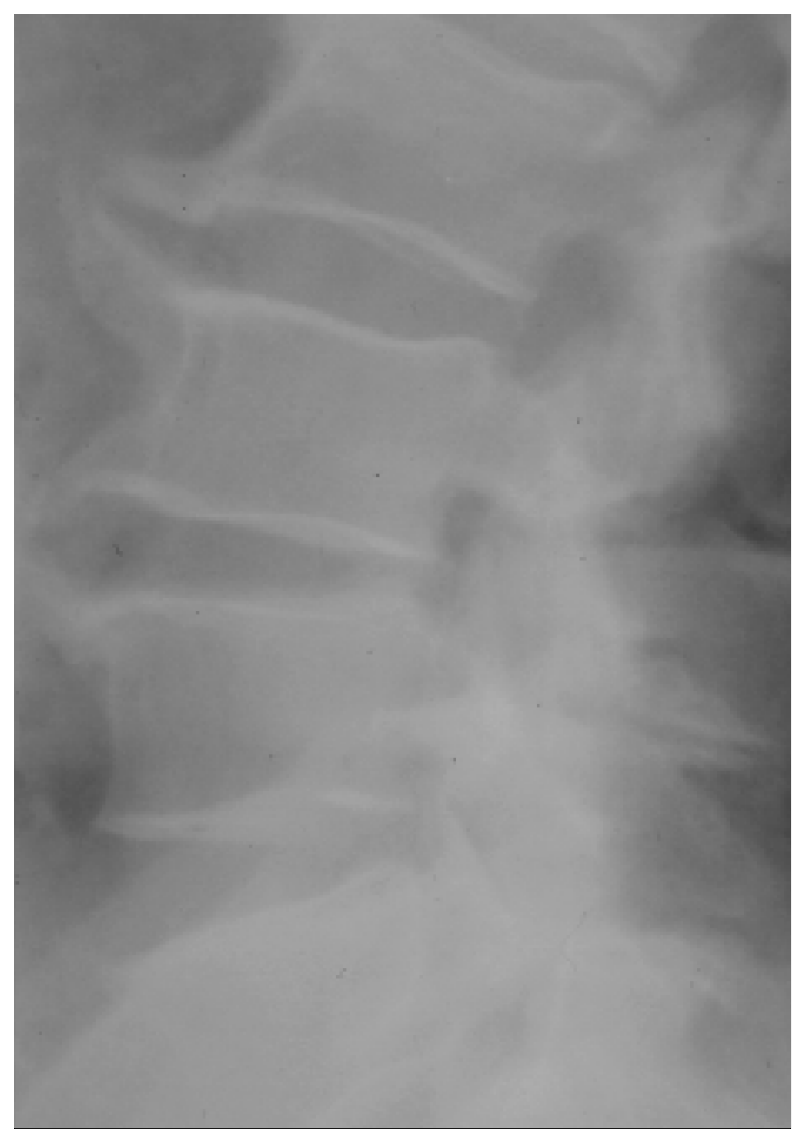

Figura 3

Típica imagen en "llama de bujía" en columna lumbar. 


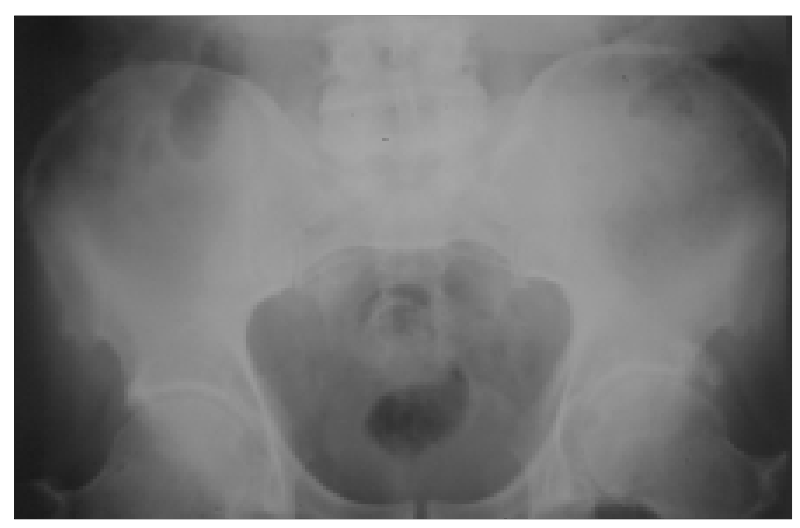

Figura 4 Hiperostosis en región pélvica.

La clínica del paciente y las características de las imágenes radiológicas nos llevaron al diagnóstico de DISH.

\section{DISCUSIÓN}

La hiperostosis es más frecuente en hombres (2:1). Entre un $25-50 \%$ de los casos presentan diabetes o un test de tolerancia oral a la glucosa patológico. También en series de diabéticos se da un $30 \%$ de casos de hiperostosis ${ }^{3,4}$.

La coincidencia de dichas patologías se podría explicar por la constitución obesa del $40 \%$ de los pacientes hiperostósicos y por su edad avanzada, características que hacen más frecuente la diabetes.

También se halla en un $58 \%$ de gotosos, que acostumbran a presentar como factor asociado la obesidad $^{3}$.

\section{La radiología}

La imagen radiológica es la tarjeta de visita de la enfermedad hiperostósica y a menudo un hallazgo espectacular 5 .

La osificación se inicia en la cara anterior o lateral del cuerpo vertebral o bien en la zona más periférica del anillo, formando un triángulo de base externa. Posteriormente se forma una osificación contigua que cubre la cara anterior y el disco.

Resnick propone tres criterios diagnósticos ${ }^{6}$ :

1. La presencia de osificaciones exuberantes en la cara anterolateral de al menos 4 vértebras contiguas.

2. La presencia de una relativa preservación del espacio intervertebral en el segmento afecto y la ausencia de cambios degenerativos en el disco (fenómeno de vacío o esclerosis del cuerpo vertebral).

3. La ausencia de afectación de las articulaciones sacroilíacas (erosiones, esclerosis, disminución del espacio intraarticular).

Los dos primeros criterios nos ayudarán a distinguirla de la espondiloartrosis y el tercero de la es- pondilitis anquilosante. Sin embargo en pacientes ancianos pueden coexistir ambas patologías (hiperostosis) y patología degenerativa (espondiloartrosis), que dificultará el diagnóstico diferencial.

\section{Alteraciones}

\section{Región dorsal}

La hiperostosis predomina en la región dorsal y media baja ( $7^{\mathrm{a}}-11^{\mathrm{a}}$ vértebra dorsal $)^{7}$ (Fig. 2).

Aunque la osificación puede afectar tanto al lado derecho como al izquierdo del cuerpo vertebral, es más común en el lado derecho (presumiblemente por un efecto inhibidor del crecimiento óseo por la aorta torácica) ${ }^{6}$.

También podemos observar áreas radiolucentes dentro de la osificación ósea a nivel de disco intervertebral.

\section{Región cervical}

Las alteraciones a nivel cervical son frecuentes en la hiperostosis predominando entre la $4^{\mathrm{a}}-7^{\mathrm{a}}$ vértebra cervical. El caso clínico muestra una afectación avanzada en forma de bloque completo (Fig. 1).

Aunque lo más frecuente es que la osificación se localice en la cara antero-lateral del cuerpo vertebral, en esta región no es infrecuente la afectación de la cara posterior del cuerpo o ligamento longitudinal posterior.

\section{Región lumbar}

La presentación más frecuente es en forma de osificaciones exuberantes que parten de la zona próxima al ángulo antero-superior del cuerpo vertebral (imagen en "llama de bujía") (Fig. 3). La osificación puede tener un grosor entre 1 y $20 \mathrm{~mm}$.

\section{Alteraciones radiológicas extraespinales}

Son muy frecuentes y características. Aunque pueden presentarse en cualquier localización, aparecen típicamente en pelvis, cadera, calcáneo, y rodilla ${ }^{2}$.

En la pelvis se hallan alteraciones hiperostósicas en casi todos los casos (70\%). Se puede observar: aumento de la densidad de las crestas iliacas, periostosis irregular de los bordes ilíacos y del isquión, osificación de los ligamentos iliolumbares y sacroisquiáticos puentes óseos en el extremo inferior de las sacroilíacas o en la parte superior de la sínfisis púbica.

En la cadera se puede observar una osificación acetabular que se distingue de los osteofitos de la coxartrosis por su base ancha de implantación y su 
forma grosera, cuadrangular o triangular. Lo más habitual es que se trate de una proliferación cotiloidea supra-externa o infero-interna de base ancha y bordes irregulares. En todo caso la interlínea articular estará conservada (Fig. 4).

También existen casos en que coexiste con una artrosis y observamos un pinzamiento de la interlínea articular que nos dificultará el diagnóstico. No debemos olvidar la frecuente coexistencia de ambas patologías propias del anciano.

En las articulaciones periféricas las formaciones hiperostósicas son menos frecuentes. Mencionaremos la hiperostosis de la rótula que aparece en la cara anterior (en la inserción del cuádriceps) a diferencia de la osteofitosis de la gonartrosis que asienta en la cara femoral; y la hiperostosis que aparece en la cara posterior del calcáneo y en la inserción del tríceps en el olécranon.

\section{Manifestaciones clínicas}

La hiperostosis ha sido considerada una entidad radiólogica cuyas manifestaciones clínicas son de poca importancia ${ }^{6}$. En general es verdad, aunque al tratarse de una patología propia del anciano puede verse enmascarada por otras patologías degenerativas. Resnick afirma que la mayoría de pacientes tienen síntomas propios de hiperostosis ${ }^{6}$.

\section{Manifestaciones clínicas vertebrales}

La más frecuente es la limitación de la movilidad vertebral. Siempre que hallemos una limitación de la movilidad en un anciano que no refiere dolor, debemos pensar en la posibilidad de una hiperostosis. Los pacientes hiperostósicos, padecen dorsalgias, cervicalgias o lumbalgias con una frecuencia superior ${ }^{8}$.

En algunos casos pueden aparecer episodios agudos dolorosos e intensos, en particular en la región dorsal; pensaremos entonces en el síndrome por sobrecarga dinámica del segmento móvil que se localiza en discos no afectados por la hiperostosis pero situados junto a zonas vertebrales anquilosadas, lo que sugiere que se trata de procesos discales degenerativos favorecidos por la sobrecarga dinámica que representa ser el único segmento móvil en una columna anquilosada.

\section{Síndromes por ocupación de espacio}

\section{Mielocompresivos}

La osificación del ligamento longitudinal posterior y del ligamento amarillo puede producir síndromes mielocompresivos en la región cervical.
Es la segunda causa más frecuente tras la espondiloartrosis ${ }^{8}$. El diagnóstico diferencial entre ambos procesos será muy importante ya que si se recurre a la intervención, el abordaje será distinto.

\section{Disfagia}

La hiperostosis situada en la cara anterior de los cuerpos vertebrales puede comprimir el esófago produciendo disfagia en las zonas próximas a los puntos de inserción (en el cartílago cricoides y el hiato diafragmático $)^{8}$.

El cuadro clínico es muy variable. En este caso la técnica quirúrgica también variará si se trata de una disfagia producida por una hiperostosis o por un osteofito artrósico.

\section{Manifestaciones clínicas en miembros}

La hiperostosis en los miembros con excepción de las caderas, produce poca sintomatología clínica. Las caderas presentan formaciones hiperostósicas en el $72 \%$ de los $\operatorname{casos}^{6}$, que se suelen manifestar como una ligera limitación de la movilidad y sólo en un $15 \%$ serán dolorosas.

En los pacientes en que la presencia de la hiperostosis contraindica, aunque no de forma absoluta, la prótesis total de cadera por la gran tendencia a desarrollar osificaciones alrededor de la prótesis ${ }^{8,9}$.

La talalgia por espolones hiperostósicos en el calcáneo es otra manifestación clínica frecuente y en ocasiones requiere la extirpación quirúrgica.

\section{Tratamiento y pronóstico}

La hiperostosis es considerada una entidad de pronóstico benigno.

El médico de familia deberá tener una actitud expectante ante las posibles complicaciones ya descritas de la enfermedad.

El síndrome por sobrecarga dinámica del segmento móvil se tratará con analgésicos y/o antiinflamatorios. El síndrome mielocompresivo y la disfagia tendrán un abordaje quirúrgico, derivando al paciente al segundo nivel asistencial. Respecto a la coxopatía hiperostósica tendremos presente que la prótesis total de cadera está contraindicada aunque sólo de forma relativa.

\section{Papel del médico de familia}

La hiperostosis es un hallazgo radiológico muy frecuente en nuestras consultas, aunque en ocasiones no es bien conocida o se confunde con otras patologías osteoarticulares degenerativas. 
El diagnóstico de la enfermedad es sencillo y accesible ya que sólo requiere la práctica de una radiografía simple.

El diagnóstico diferencial se deberá establecer con la espondiloartrosis y la espondilitis anquilosante.

El seguimiento del paciente se centrará en detectar precozmente las posibles complicaciones.
La información al paciente sobre su pronóstico benigno es fundamental.

CORRESPONDENCIA:

Avelina Espina Castilla

C/ Vélez Rubio s/n

08918 Badalona. Barcelona

\section{Bibliografía}

1. Forestier J, Rotès-Querol J. Senile ankylosing hiperostosis of the spine. Ann Rheum Dis 1950; 9: 321-30.

2. Resnick D, Shaul SR, Robins JM. Diffuse idiopathic skeletal hyperostosis (DISH): Forestier's disease with extraespinal manifestations. Radiology 1975; 115: 513-24.

3. Rotés-Querol J. Hiperostosis anquilosante vertebral (HAV). Diffuse idiopathic skeletal hyperostosis (DISH). MTA-Medicina Interna 1987; 5 (2): 72-103.

4. Larrosa Padró M. Limitación de la movilidad articular en la diabetes mellitus. Rev Esp Reumat 1995; 22: 289-95.

5. Resnick D, Curd J, Shapiro RF, Wiesner KB. Radiographic abnormalities of rheumatoid arthritis in patients with diffuse idiopathic skeletal hyperostosis. Arthritis Rheum 1978; 21: 1 .

6. Resnick D. Diffuse idiopathic skeletal hyperostosis. In: Bone and joint imaging. Resnick D. Philadelphia: Saunders Co. 1989; 440-51.

7. Resnick D, Niwayama G. Radiographic and pathologic features of spinal involment in difusse idiopathic skeletal hyperostosis (DISH). Radiology 1976; 119: 559.

8. Rotés-Querol J. Hiperostosis anquilosante vertebral (hiperosotosis esquelética difusa idiopática). En: Andreu Sánchez JL, Barceló Garcia M, Herrero-Beaumont Cuenca G, Martín Mola E, Olivé Marqués A. Manual de enfermedades reumáticas de la Sociedad Española de Reumatología. Madrid: Mosby/Doyma S.A., 1996; 797-802.

9. Rotés-Querol J. Hiperostosis anquilosante vertebral. En: Pascual Gómez E, Rodríguez Valverde V, Carbonell Abelló J, Gómez-Reino Carnota JJ. Tratado de Reumatología. Tomo II. Madrid: Arán Ediciones, S.A., 1998; 1697-711. 